\title{
LA FORMACIÓN Y ADQUISICIÓN DE CONOCIMIENTO PARA ENTRENAR Y DIRIGIR DE LOS ENTRENADORES DEL FÚTBOL ÉLITE DE COSTA RICA
}

Rivas-Borbón, Oscar Milton; Víquez-Ulate, Fabián; Revuelta-Sánchez, Inés; Salas-Cabrera, Jorge; Gutiérrez-Vargas, Juan Carlos

LA FORMACIÓN Y ADQUISICIÓN DE CONOCIMIENTO PARA ENTRENAR Y DIRIGIR DE LOS

ENTRENADORES DEL FÚTBOL ÉLITE DE COSTA RICA

MHSalud, vol. 15, núm. 2, 2018

Universidad Nacional, Costa Rica

Disponible en: http://www.redalyc.org/articulo.oa?id=237056017002

DOl: https://doi.org/10.15359/mhs.15-2.1

Esta obra está bajo una Licencia Creative Commons Atribución-NoComercial-SinDerivar 3.0 Internacional. 


\section{LA FORMACIÓN Y ADQUISICIÓN DE CONOCIMIENTO PARA ENTRENAR Y DIRIGIR DE LOS ENTRENADORES DEL FÚTBOL ÉLITE DE COSTA RICA}

\section{THE FORMATION AND ACQUISITION OF KNOWLEDGE OF THE ELITE SOCCER COACHES OF COSTA RICA TO TRAIN AND COACH}

\section{A FORMAÇAO E A AQUISIÇÃO DO CONHECIMENTO PARA TREINAR E LIDERAR OS TREINADORES DO FUTEBOL DE PRIMEIRA DIVISAO DA COSTA RICA}

Oscar Milton Rivas-Borbón

Universidad Nacional, Costa Rica

milton.rivas.borbon@una.cr

iD http://orcid.org/0000-0001-6304-5159

Fabián Viquez-Ulate

Universidad Nacional, Costa Rica

favz13@gmail.com

(D) http://orcid.org/0000-0003-4980-3464

Inés Revuelta-Sánchez

Universidad Nacional, Costa Rica

inesrevuelta@gmail.com

(iD http://orcid.org/0000-0003-4560-1953

Jorge Salas-Cabrera

Universidad Nacional, Costa Rica

josaca10@hotmail.com

(iD http://orcid.org/0000-0002-9226-3144

Juan Carlos Gutiérrez-Vargas

Universidad Nacional, Costa Rica

jucagu@msn.com

(iD) http://orcid.org/0000-0002-0689-6771
DOI: https://doi.org/10.15359/mhs.15-2.1

Redalyc: http://www.redalyc.org/articulo.oa? $\mathrm{id}=237056017002$

Recepción: 03 Mayo 2018

Aprobación: 27 Julio 2018

\section{Resumen:}

El objetivo de este estudio fue explorar aspectos relacionados con la formación y las fuentes de conocimiento que hoy en día poseen los entrenadores de futbol élite costarricense, tanto para dirigir como para entrenar, y que ejercen influencias en la toma de sus decisiones de su entorno deportivo. Para ello, se ha realizado una investigación cualitativa, en la cual la recolección de los datos se hizo a través del método denominado triangulación metodológica. Este consistió en la aplicación de 2 herramientas metodológicas, la entrevista y el cuestionario, cuyos resultados al final fueron comparados entre sí. La población del estudio fue de 10 entrenadores de la liga de primera división del fútbol costarricense. Los resultados indicaron que los entrenadores basan su labor de dirigir y entrenar en casi todas las subcategorías de adquisición de conocimientos que se plantearon en el estudio; sin embargo, las subcategorías de experiencia como jugador, experiencia profesional y el manejo de herramientas tecnológicas fueron a las que le asignaron mayor valor, sobre todo para el ámbito de dirigir en la competencia. $\mathrm{Al}$ mismo tiempo, se encuentra que la subcategoría interacción entre colegas fue a la que le asignaronn el menor valor. Las conclusiones del estudio deberían ser tomadas en consideración a la hora del diseño curricular en la formación de entrenadores de fútbol.

Palabras Clave: formación, conocimiento, entrenadores, fútbol.

\section{ABSTRACT:}

This study aimed to explore the aspects related to the formation and sources of knowledge that Costa Rican elite soccer coaches have in order to coach and train, and how those aspects influence the decision-making process of soccer coaches' in their sport 
environment. For this purpose, a qualitative research project has been carried out, where data was collected through the method specifically known as methodological triangulation, which consisted in the application of two methodological tools, the interview and the questionnaire, whose final results were compared with each other. The study population consisted of 10 coaches of the Costa Rican First Division Soccer League. The results indicate that coaches base their work of coaching and training on almost all subcategories of acquisition of knowledge that were raised in the study. However, the subcategories of experience as a player, professional experience, and the use of technological tools were assigned a greater value, especially regarding the field of coaching in competition. At the same time, the subcategory interaction between colleagues was assigned the lowest value. The conclusions of the study should be analyzed when designing curricula in the training of soccer coaches.

KEYWORDS: formation, knowledge, coaches, soccer.

\section{RESUMo:}

O objetivo deste estudo foi explorar aspectos relacionados com a capacitação e às fontes de conhecimento que os técnicos de futebol de primeira divisão da Costa Rica possuem atualmente, tanto para dirigir e treinar, quanto para exercer influências na tomada de decisóes no seu ambiente desportivo. Para tanto, realizou-se uma pesquisa qualitativa, na qual a coleta de informações foi realizada por meio do método conhecido como triangulação metodológica, que consistiu na aplicação de dois instrumentos metodológicos, a entrevista e o questionário, cujos resultados foram comparados ao final. A população do estudo foi de 10 treinadores da primeira divisão do futebol costa-riquenho. Os resultados indicaram que os treinadores baseiam seu trabalho de dirigir e treinar, em quase todas as subcategorias de aquisição de conhecimento que foram levantadas no estudo, no entanto, as subcategorias de experiência como jogador, experiência profissional e uso de ferramentas tecnológicas foram as que mais atribuíram valor, especialmente ao campo de dirigir para competência. Ao mesmo tempo, verifica-se que a subcategoria interação entre os colegas recebeu o menor valor. As conclusões do estudo devem ser levadas em consideração ao projetar currículos na capacitação de treinadores de futebol. Palavras-ChaVE: Treinamento, Conhecimento, Treinadores, Futebol.

\section{INTRODUCCIÓN}

El proceso de dirigir y entrenar está fundamentalmente basado en procedimientos cognitivos que conducen a la toma de decisiones estratégicas deportivas en todas sus dimensiones. Sin embargo, estos métodos mentales de dirigir, como p. ej. la "lectura de un juego competitivo" o el de entrenar, como p. ej. la planificación de los entrenamientos, son poco conocidos (Castillo, Ramis, Cruz y Balaguer, 2015).

Un estudio realizado por Lemyre et al. (2007) en entrenadores de hockey sobre hielo, fútbol y baseball, así como otro de Jiménez-Saiz, Gómez-Ruano, Borrás-Luján, y Calvo (2007) en entrenadores de baloncesto concluyeron que el aprendizaje adquirido para llegar a ser entrenador deportivo de élite es un proceso complejo desarrollado sin un patrón específico. Por tanto, la formación de los entrenadores no debe estar supeditada exclusivamente a cursos formales. También, agregan que los programas formales son una de las muchas oportunidades para aprender a entrenar y dirigir, al igual que existen otras tales como las experiencias previas en calidad de jugadores o asistentes; la interacción con otros colegas, y, por supuesto, las propias vivencias de aprendizaje presentadas en la competencia y cuyas reflexiones, alrededor de ellas mismas, proveen enseñanzas importantes.

Por lo dicho, ahondar en el origen y la comprensión de estos procesos mentales podría aportar información fundamental a la hora de elaborar los cursos de formación dirigidos a entrenadores, sobre todo en el ámbito de la dirección de equipo (coaching), pues se vendría a incrementar el acervo cognitivo de aquellos, en relación con su capacidad para identificar no solo los elementos o factores decisivos que interactúan a lo interno del proceso de entrenamiento y competición, sino también con su capacidad para integrarlos en la toma de decisiones de su entorno deportivo. Sobre este tema, los entrenadores mencionan que, al recibir un taller sobre coaching, se sienten preparados para integrarlo a sus prácticas y partidos; además, manifiestan que desarrollan la capacidad (coaching) de empoderamiento hacia sus equipos (Castillo, Ramis, Cruz y Balaguer, 2015).

También, se puede considerar que la comunicación entre jugadores y entrenadores en el proceso de coaching es importante, debido a que esta permite mejorar la destreza del entrenador para la aplicación 
de planteamientos tácticos. Incluso, esta comunicación genera que los entrenadores sean valorados como sinceros, seguros de sí mismos, honestos, inteligentes y motivadores (Rosado, Palma, Mesquita y Moreno, 2007).

Ahora bien, en relación con la adquisición de conocimientos de los entrenadores, Cushion, Armour y Jones (2012) argumentan que la experiencia de estos profesionales y la observación de otros colegas se ubica entre sus principales fuentes de conocimiento. Además, mucho de lo que aprenden es a través de cursos prácticos y de la interacción con su contexto de entrenamiento y competición, así como de una variedad de fuentes informales de conocimiento.

Igualmente, en cuanto al escenario del aprendizaje de los entrenadores, investigaciones realizadas por Erickson, Bruner, MacDonald y Coté (2008); Pérez (2002), y Feu-Molina, Ibáñez-Godoy, Lorenzo-Calvo, Jiménez-Saiz y Cañadas-Alonso (2012) manifiestan que tal escenario se da a través de la experiencia (educación informal), lo cual regularmente se destaca como un componente clave para el entrenador y su formación, sobre todo después de tener muchos años de ejercer la profesión. El proceso de reflexión que conlleva la experiencia se ha identificado como la teoría de aprendizaje central y ha sido traducido a la literatura sobre coaching como un mecanismo a través del cual esa experiencia produce aprendizaje para el entrenador.

La eficacia del entrenador para tomar decisiones, tanto en el nivel competitivo como en el de entrenamiento, pareciera depender de muchos factores; sin embargo, el hecho de que estas sean acertadas durante el proceso de entrenamiento y de competición se basa, primordialmente, en procesos informales de educación que subyacen en el entrenador, es decir, en situaciones de aprendizaje empíricas (Cushion et al., 2012; Nasch y Collins, 2006; Lemyre et al., 2007; Ayala-Zuluaga, Aguirre-Loaiza y Ramos-Bermúdez, 2015; Werthner y Trudel, 2006). Es así como la experiencia profesional es considerada no solo una de las fuentes de información más importantes para el desarrollo del entrenador deportivo, sino también uno de los requisitos para que este sea considerado un experto (Abraham, Collins y Martindale, 2006; Nash y Collins, 2006; Erickson et al., 2008; Pérez, 2002; Feu-Molina, Ibáñez-Godoy, Lorenzo-Calvo, Jiménez-Saiz y Cañadas-Alonso, 2012).

A pesar de lo anterior, no se puede dejar de lado la adquisición de conocimientos a través de la formación académica, pues su aporte es relevante en lo que respecta a la formación profesional del entrenador, ya que los saberes adquiridos sirven como bases teóricas y prácticas para una formación especializada (Coté, Salmela, Trudel, Baria y Russel, 1995; Vallée y Bloom, 2005; Abraham, Collins y Martindale, 2006; Coté, Young, North y Duffy, 2007; Ayala-Zuluaga, Aguirre-Loaiza y Ramos-Bermúdez, 2015; Vinicia, Mayorga, Ruiz y Blanco, 2016).

En referencia a la educación formal e informal de los entrenadores, los investigadores Barros, Ribeiro, Arroyo, Rosado, Sousa y Pereira (2010), al igual que Pérez (2002) concluyeron, en su estudio, que los entrenadores con formación académica en educación física y deportes suelen presentar niveles superiores de competencia en las actividades ligadas directamente al entrenamiento, en lo cual destacan la planificación anual y plurianual, la dirección y formación de entrenadores principiantes y las competencias pedagógicodidácticas. En cambio, los entrenadores con más experiencia se perciben más competentes en planificación, evaluación y conducción de los entrenamientos, así como en la dirección y formación de deportistas y entrenadores principiantes.

A diferencia de lo expuesto anteriormente, en Costa Rica se carece de evidencia científica que indique el dónde y el cómo los entrenadores del fútbol élite adquirieron sus conocimientos, tanto para dirigir como para entrenar, razón por la cual se ha planteado este estudio, con el propósito de caracterizar aspectos alrededor de la formación de dichos entrenadores y, a su vez, determinar las posibles fuentes de conocimientos que utilizan para ejercer su labor profesional. 


\section{Metodología}

La metodología empleada en la investigación es cualitativa y se usó el método de triangulación descrito por Okuda y Gómez (2005), específicamente, se utilizó la triangulación metodológica, con el fin de darle mayor validez y calidad al estudio. Esta consiste en la posibilidad de usar varios métodos, tanto cualitativos como cuantitativos. Se supone que, al utilizar un solo método, el estudio es más vulnerable a sesgos y fallas metodológicas inherentes a cada método y que la triangulación metodológica ofrece la alternativa de poder visualizar un problema desde diferentes ángulos; de esta manera, se aumenta la validez y consistencia de los hallazgos. Este término metafórico de triangulación busca patrones de convergencia para desarrollar

o corroborar una interpretación global del fenómeno humano objeto de investigación —en este caso, la formación y el dónde adquirieron los conocimientos los entrenadores, lo cual no significa que literalmente se tengan que utilizar 3 métodos-. Una de las principales ventajas de la triangulación metodológica es que, cuando los métodos utilizados proyectan resultados similares, corroboran y brindan mayor fuerza a los hallazgos, pero cuando, por el contrario, aquellos resultados no lo hacen, la triangulación ofrece una oportunidad para que se elabore una perspectiva más amplia en cuanto a la interpretación del fenómeno en estudio. Por esto la combinación de métodos cuantitativos y cualitativos ofrece la visión de los diferentes aspectos del fenómeno estudiado, más que la repetibilidad de los hallazgos (Okuda y Gómez, 2005).

Particularmente, para la investigación se desarrollaron 2 estrategias metodológicas, una cualitativa y otra cuantitativa, que corresponden a la triangulación metodológica planteada por Okuda y Gómez (2005) ya aludida. La primera consistió en realizar una entrevista semiestructurada y la segunda, un cuestionario. Ambos instrumentos fueron orientados a indagar cómo y dónde adquirieron los entrenadores el conocimiento para entrenar y dirigir.

\section{Categorías y subcategorías de conocimiento}

Las categorías y subcategorías de conocimiento que se establecieron para la investigación fueron determinadas con base en otros estudios similares a este, como los realizados por Jiménez-Sáiz, LorenzoCalvo, Gómez-Ruano y Lorenzo-Calvo (2009) y Cushion, Armour y Jones (2012), fundamentados en la experiencia obtenida por los investigadores en este campo profesional. Al final, se determinaron 3 grandes categorías de conocimiento: la educación informal, la educación formal y los recursos tecnológicos.

Referente a la categoría educación informal, se subdividió en 8 subcategorías de conocimiento, a decir:

1. Experiencia profesional. Todos aquellos conocimientos adquiridos en las experiencias que conlleva el propio ejercicio de su profesión.

2. Experiencia como jugador. Conocimientos basados en las experiencias que adquirieron los técnicos a través de su vida como jugadores profesionales.

3. Experiencia como asistente. Conocimientos sustentados en las experiencias cognitivas adquiridas como asistentes técnicos en equipos profesionales.

4. Estudios independientes. Se trata de aquellos conocimientos adquiridos a través de la exploración autodidacta, como la lectura de libros, revistas especializadas, artículos científicos, otros.

5. Relación con sus jugadores. Obedece a los conocimientos adquiridos a través de su comunicación con sus dirigidos.

6. Relación con su cuerpo técnico. Conocimientos adquiridos a través de su comunicación con sus asistentes técnicos y preparador físico.

7. Mentores. Está relacionada con los exentrenadores que tuvieron en su carrera como futbolistas y que, de una u otra manera, determinaron sus conocimientos para dirigir y entrenar. 
8. Interacción entre colegas. Subcategoría que se sustenta en la posible adquisición de conocimientos a través de la interacción comunicativa de experiencias y conocimientos entre colegas.

La categoría de conocimiento educación formal consistió en la participación de los entrenadores en procesos oficiales o formales de formación como a) la obtención de conocimientos a través de estudios universitarios con grado académico y b) conocimientos adquiridos a través de escenarios educativos que aquí se han denominado como educación continua; entre ellos, congresos, cursos libres, simposios, pasantías, entre otros.

La última clasificación de categoría de adquisición de conocimientos fueron los recursos tecnológicos, referida a la utilización de herramientas tecnológicas como fuente de conocimiento: videos, software específico, GPS, entre otros.

\section{Muestra de expertos}

Se toma toda la población de entrenadores que estaban en equipos o los habían dirigido en un nivel profesional o de selecciones nacionales mayores o menores. De esta totalidad de entrenadores, se seleccionaron para el estudio aquellos que cumplieran con los siguientes 2 requisitos o criterios: a) tener al menos 5 años de experiencia como entrenador de fútbol en primera división o con selecciones nacionales; $b$ ) que no tuviese más de 6 meses sin dirigir. Al final se conformó la lista de entrenadores que cumplían con estos requisitos, en total quedaron 12 , de los cuales 10 fueron participantes del estudio, mientras que los otros 2 no pudieron participar en él, por razones que escaparon al control de los investigadores.

Ahora bien, con la lista de entrenadores ya establecida, así como con los instrumentos o métodos de medición del estudio, se procedió a su aplicación y a confrontar los resultados obtenidos en ambos, con el propósito de observar posibles similitudes o diferencias.

\section{La entrevista y el cuestionario}

El instrumento cualitativo utilizado para obtener los datos fue la entrevista semiestructurada, mientras que el cuantitativo fue el cuestionario, también semiestructurado (Patton, 2002). El diseño de la entrevista y del cuestionario se realizaron siguiendo las siguientes fases: 1) análisis de otras entrevistas y cuestionarios; 2) diseño de la primera versión de la entrevista y del cuestionario; 3 ) estudio piloto previo; 4) versión oficial de la entrevista y del cuestionario.

\section{Análisis de datos}

Previo a la aplicación del cuestionario y la entrevista para cada entrenador, se le brindo a cada uno de ellos el documento denominado "consentimiento informado", el cual leyeron y al final firmaron, aceptando así las condiciones de la investigación.

Todas las entrevistas realizadas a los entrenadores fueron transcritas literalmente. Luego se le entregó a cada investigador del estudio una copia de la transcripción de la entrevista, con la finalidad de corroborar los datos, así como para aumentar o modificar su contenido. Esta información fue de nuevo recogida para analizarla y categorizarla.

Referente al cuestionario, se aplicó previo a cada entrevista. En él se utilizó la escala de Likert (Bisquerra y Pérez-Escoda, 2015), en la cual el nivel de importancia para cada ítem (subcategoría de conocimiento) iba de manera progresiva, a saber, al número 1 le correspondió nada, al 2 poco, al 3 algo, al 4 bastante y al 5 mucho. Ya con los datos de los cuestionarios aplicados, se procedió a determinar el promedio de cada 
MHSALUD, ISSN: 1659-097X, NOVIEMBRE-DICIEMBRE, 15(2) 2018, 1-14

ítem, estableciendo así el nivel de importancia que le dieron los entrenadores a las diferentes categorías y subcategorías de conocimiento adquirido.

\section{Resultados}

A continuación, se presentan los resultados obtenidos en las diversas categorías y subcategorías de conocimientos que fueron planteadas en el estudio (Ver Tabla 1). 


\section{TABLA 1}

Resumen de los resultados obtenidos en las entrevistas, en la categoría de educación informal y las subcategorías de experiencia profesional, experiencia como jugador, experiencia como asistente, estudios independientes, relación con sus jugadores, relación con su cuerpo técnico, mentores e interacción entre colegas

\begin{tabular}{ll}
\hline & \multicolumn{1}{c}{ Categoría: educación informal } \\
\hline & \multicolumn{1}{c}{ Subcategorias } \\
\hline & $\begin{array}{l}\text { Todos expresan que la experiencia adquirida durante el tiempo que han estado dirigiendo ha sido sumamente } \\
\text { importante, pues, han mejorado paulatinamente su capacidad de dirigir, ya que afianza su idea de juego y mejora }\end{array}$ \\
& su "lectura" sobre sus rivales. Además, agregan que todo ese conocimiento que van adquiriendo partido tras \\
partido los ha llevado a obtener un mayor bagaje de experiencias para mejorar su planificación de encuentros, \\
A. Experiencia \\
profesional & asi como el control de variables externas que podrian influenciar el rendimiento de sus equipos, tales como el \\
clima, ambientes hostiles, terreno de juego, entre otros.
\end{tabular}

Sobre la importancia de su experiencia al estar continuamente dirigiendo, uno de ellos expreso "... estos 10 años que tengo de ser entrenador he cambiado mucho mi comportamiento en la cancha, antes era más impulsivo y atrevido, ahora soy más lider por convicción que por autoridad...".

Todos los participantes del estudio, en su momento, practicaron fútbol profesional, tanto en el nivel nacional como en el internacional. Todos aducen que esa experiencia como futbolistas profesionales les benefició para ir adquiriendo conocimientos, que posteriormente fueron aplicando en su carrera como entrenadores.

Entre los aspectos que más se mencionan como medio de adquisición de conocimiento, están el manejo de las relaciones interpersonales aplicado a la dirección de sus equipos y algunos ejercicios prácticos de entrenamiento.

B. Experiencia como jugador

C. Experiencia como asistente

D. Estudios independientes

E. Relación con sus jugadore

F. Relación con su cuerpo técnico

\section{G. Mentores}

H. Interacción entre colegas

Así mismo, todos manifestaron que el hecho de haber sido exfutbolistas les desarrolló entendimiento en la interpretación del sentir de los jugadores, en diversas situaciones deportivas, como el sentimiento de derrota o de victoria, lesiones, expulsiones, conflictos grupales, entre otros. También, acotaron la adquisición de conocimientos que les han ayudado para dirigir en la competencia.

Sobre este tema, un entrenador expresó: "... mi parte universitaria es haber jugado y ahi haber acumulado conocimientos desde mis inicios como jugador...”.

6 de los 10 entrevistados tuvieron la experiencia de ser asistentes técnicos.

Los 6 entrenadores manifestaron que el hecho de haber sido asistentes les habia ayudado de diferentes maneras para su futuro en esta profesión, ya que adquirieron conocimientos para:

- La planificación y elaboración de tareas prácticas de entrenamiento.

- Ir ideando su propio estilo de juego.

- Poder desarrollar habilidades en el análisis de video y el análisis estadístico.

- Adquirir saberes de las experiencias obtenidas por los otros directores técnicos de los cuales fueron asistentes.

Sobre la adquisición de conocimiento en este ámbito, un entrenador manifestó: “... me ayudó en el ámbito de entrenamiento y dirección técnica, además de todo lo que esto conlleva...".

Todos los entrenadores mencionaron la importancia que tienen para ellos los estudios informales y su aplicación en el ámbito del entrenamiento y de la dirección. Ellos expresan que buscan información en libros, videos, blogs, páginas web deportivas, entre otras fuentes, y tratan de trasladarla a la práctica.

"... los libros cuando era adolescente fueron muy importantes para mi, ya que de ahi pude poner en práctica los contenidos del libro cuando en ese entonces entrenaba el equipo del pueblo...".

Solamente 2 entrenadores afirmaron que la relación comunicativa entre ellos y sus jugadores les ha proporcionado conocimiento en su labor. Uno de ellos manifestó que su comunicación con los jugadores no es directa, ya que "... a veces como técnico no le llego a ciertos jugadores, entonces los asistentes son los que me ayudan....". Otro entrenador comentó que, gracias a una conversación con uno de sus jugadores, él le manifestó “... los jugadores juegan presionados, porque usted entrena muy perfecto y nadie quiere fallar..."; como consecuencia de este comentario dicho entrenador, desde entonces, les menciona a sus jugadores que apliquen las herramientas que él les brinda en los entrenamientos, para que puedan utilizarlas en la competencia sin presión.

Los demás entrenadores del estudio manifestaron que, en pocas ocasiones, recurren a los futbolistas para consultarles aspectos relacionados con la ejecución de entrenamientos y planteamientos de partidos.

La interacción que tienen los entrenadores con sus respectivos asistentes es de mucha importancia para ellos, pues todos manifestaron estar siempre anuentes a los comentarios por parte de estos. Además, 3 entrenadores afirmaron que tienen reuniones muy seguidas con sus asistentes, para encontrar fortalezas y debilidades de sus equipos; sobre ello, uno expresó: ".... soy una persona que me gusta escuchar mucho mis asistentes porque si los tengo es para que ayuden ....".

Así mismo, pocos entrenadores expresaron que en los inicios de su carrera no tomaban mucho en cuenta a sus asistentes en los aspectos relacionados con el rendimiento deportivo del equipo. Sin embargo, con el avance del tiempo, aprendieron que estos pueden ser útiles.

Todos los entrenadores del estudio manifestaron que en su trayectoria profesional como jugadores han tomado tanto los aspectos positivos como aquellos negativos de sus ex entrenadores. Sobre esto, uno de ellos manifestó: "... tuve entrenadores que me enseñaron a cómo no hacer las cosas...", y otro mencionó: "... tuve un entrenador que tácticamente era muy bueno...".

De los 10 entrenadores analizados, 3 aseveraron que el diálogo, con sus colegas, ha tenido un efecto positivo en la adquisición de conocimiento para entrenar y dirigir. No obstante, el resto de los entrenadores manifestó que no mantienen una relación tal, que les permita un intercambio técnico de conocimientos con sus colegas. Por ejemplo, uno de ellos adujo: "... hay un concepto de esconder información, hay celos y cuesta mucho entablar relación con otros técnicos...". 
La Tabla 1 muestra que los entrenadores entrevistados le otorgaron mucha importancia a 7 de las 8 subcategorías, como medios de adquirir los conocimientos para entrenar y, sobre todo, para dirigir sus equipos. No obstante, en la subcategoría de interacción entre colegas, 7 de los 10 entrevistados manifestaron no adquirir conocimiento directo de parte de sus colegas.

TABLA 2

Resumen de los resultados obtenidos en las entrevistas, en la categoría de conocimientos de educación formal y la subcategoría de educación continua

\begin{tabular}{cl}
\hline \multicolumn{1}{c}{ Categoría: educación formal } \\
\hline & \multicolumn{1}{c}{ Subcategoría } \\
\hline & $\begin{array}{l}\text { La totalidad de los entrenadores reconoce que a través de la educación } \\
\text { formal obtuvieron conocimientos que les han beneficiado en la pla- } \\
\text { nificación, organización y estructuración de los entrenamientos. Sin } \\
\text { embargo, solamente } 3 \text { de ellos reconocieron que dichos conocimien- }\end{array}$ \\
& $\begin{array}{l}\text { tos adquiridos en la academia les habían ayudado a dirigir partidos. } \\
\text { Por otro lado, 2 de los entrenadores entrevistados obtuvieron un grado } \\
\text { universitario de Diplomado en dirección técnica en fútbol. }\end{array}$ \\
& $\begin{array}{l}\text { Sobre la adquisición de conocimientos, un entrenador expresó: "La } \\
\text { educación formal me ayudó a la planificación y organización de entre- } \\
\text { namientos, pero en la dirección de los partidos no...". }\end{array}$ \\
\hline
\end{tabular}

En la Tabla 2, se muestra que el conocimiento que han adquirido los entrenadores a través de la educación formal (en carrera universitaria, cursos libres, congresos, simposios, pasantías, entre otros) les ha ayudado, fundamentalmente, más en su labor profesional de entrenar y, parcialmente, en la de dirigir.

TABLA 3

Resumen de los resultados obtenidos en las entrevistas, en la categoría de conocimientos de recursos tecnológicos y la subcategoría de manejo de herramientas tecnológicas

\begin{tabular}{ll}
\hline & Categoría: recursos tecnológicos \\
\hline & \multicolumn{1}{c}{ Subcategoría } \\
\hline & $\begin{array}{l}\text { Para todos los entrenadores, los recursos tecnológicos, y sobre } \\
\text { todo el análisis de video, son considerados necesarios y de uti- } \\
\text { lidad, pues con ellos pueden analizar a sus rivales, planificar } \\
\text { y establecer tanto tácticas como estrategias. En general, les } \\
\text { ayudan a tomar decisiones en los ámbitos del entrenamiento }\end{array}$ \\
$\begin{array}{l}\text { Manejo de } \\
\text { herramientas } \\
\text { tecnológicas }\end{array}$ & $\begin{array}{l}\text { Ahora bien, sobre la utilización de determinados instrumentos } \\
\text { tecnológicos, algunos técnicos manifestaron que han utilizado } \\
\text { el GPS y ciertos tipos de estadísticas específicas. Por ejemplo, } \\
\text { la gran mayoría de los entrenadores expresó la importancia } \\
\text { del análisis de video; uno de ellos mencionó que “.... nos ha } \\
\text { permitido enseñarle y mostrarle al jugador cómo ataca y cómo } \\
\text { defiende el rival...”. }\end{array}$ \\
\hline
\end{tabular}

En la Tabla 3, se puede observar que los conocimientos adquiridos por medios tecnológicos, tales como videos, GPS, software, todos los entrenadores los ubican entre las más importantes subcategorías de conocimientos, sobre todo para el ámbito de dirigir, más que para el de entrenar. 
A continuación, se presenta la Tabla 4 con los resultados del cuestionario, el cual se verá reflejado en la dimensión cuantitativa, a través del nivel de importancia que le dieron los entrenadores a las diferentes categorías y subcategorías de conocimiento adquirido tanto para dirigir como para entrenar.

TABLA 4

Matriz de los resultados de puntaje promedios, obtenidos sobre las diversas categorías y subcategorías de conocimiento que poseen los entrenadores para entrenar y dirigir

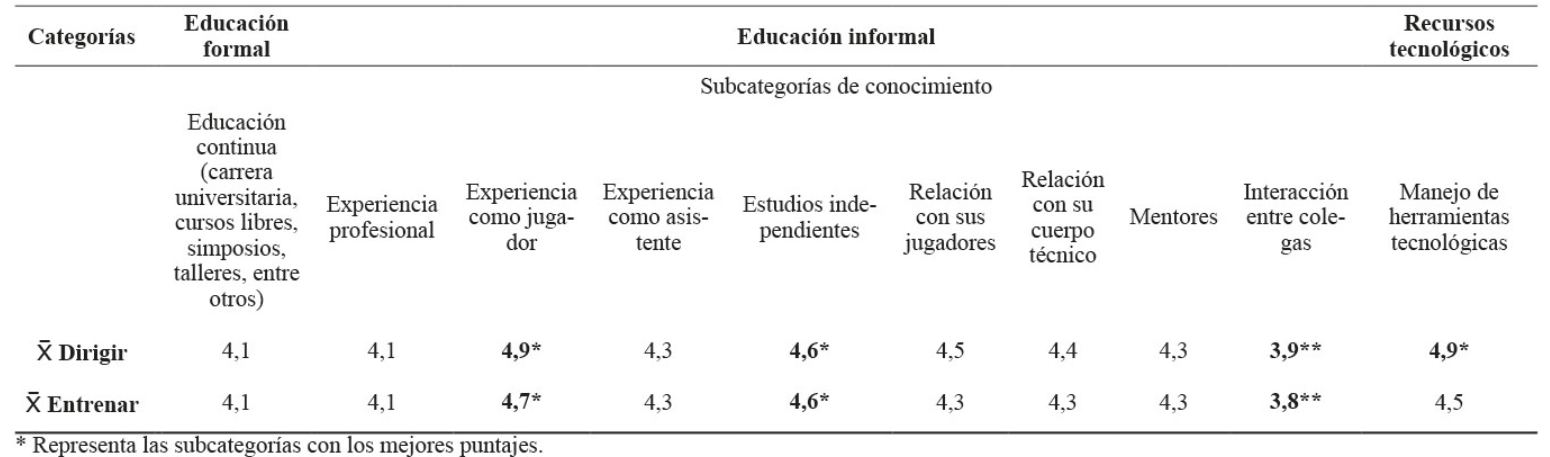

** Representa las subcategorías con los menores puntajes

En la tabla 4, se observa que los entrenadores ubicaron el manejo de herramientas tecnológicas $(4,9)$, junto con la experiencia como jugador $(4,9)$, como las fuentes de mayor adquisición de conocimiento para dirigir, mientras que la labor de entrenar también estuvo sustentada en sus experiencias como futbolistas $(4,7)$ y en los estudios independientes que realizaron $(4,6)$. Por otro lado, los entrenadores ubicaron la subcategoría de interacción entre colegas $(3,9$ y 3,8) como la de menor relevancia en la adquisición de conocimientos, tanto para dirigir como para entrenar.

\section{Discusión}

Según los resultados presentados en la Tabla 1, en la cual se dimensiona la parte cualitativa del estudio, la categoría de conocimiento denominada educación informal, se observa que los entrenadores admiten haber adquirido bastantes conocimientos en 7 de las 8 subcategorías planteadas, sobre todo de experiencia profesional, experiencia como jugador, estudios independientes y mentores, mientras que en la categoría de interacción con colegas la gran mayoría de ellos admitió no haber adquirido saberes.

Comparando los resultados anteriores con los obtenidos en la aplicación del cuestionario, se puede decir que en algunas subcategorías fueron muy similares. Por ejemplo, los ítems del cuestionario relacionado con las subcategorías de experiencia como jugador y estudios independientes obtuvieron las más altas calificaciones (ver Tabla 4).

Por tanto, los resultados derivados de la categoría de educación informal, tanto en las entrevistas como en el cuestionario, parecen orientarse a que muchos de los conocimientos que han adquirido los entrenadores investigados están sustentados en sus propias experiencias como futbolistas y en aquellas adquiridas a diario en sus labores profesionales. Estos resultados se ven respaldados por estudios como los realizados por AyalaZuluaga, Aguirre-Loaiza y Ramos-Bermúdez (2015), por Erickson, Bruner, MacDonald y Coté (2008) y por Feu-Molina, Ibáñez-Godoy, Lorenzo-Calvo, Jiménez-Saiz y Cañadas-Alonso (2012), quienes destacaron la experiencia como un componente clave para la formación del entrenador.

Prosiguiendo con el análisis de los resultados de cada subcategoría correspondiente al área de educación informal, específicamente la de la experiencia profesional, tanto en la entrevista como en el cuestionario pareciera existir una concordancia de que, en el ejercicio frecuente del entrenador, respecto de estar 
planteando entrenamientos y partidos, está implícito un aprendizaje, producto de esa constante toma de decisiones deportivas. Sobre este tema, autores como Cushion et al. (2012), Nasch y Collins (2006), Lemyre et al. (2007), Werthner y Trudel (2006) encontraron que la toma de decisiones acertadas durante el proceso de entrenamiento y competición está sustentada fundamentalmente en la educación informal y, sobre todo, en el aprendizaje empírico.

La subcategoría de conocimiento por experiencia como jugador fue altamente valorada por los entrenadores estudiados y en ambos instrumentos de medición. Todos manifestaron que el hecho de haber sido futbolistas profesionales les ha ayudado, entre otras cosas, a comprender y saber manejar diversas situaciones deportivas. Estudios realizados por Lemyre et al. (2007) y Jiménez-Saiz, Gómez-Ruano, BorrásLuján y Calvo (2007) encontraron que el aprendizaje para llegar a ser un entrenador élite es muy complejo, pero que existen fuentes importantes de conocimiento como la experiencia como jugador.

En lo que se refiere a las subcategorías de conocimientos adquiridos a través del proceso de relación o comunicación del entrenador con sus asistentes y jugadores, los resultados del estudio manifiestan que los profesionales dicen ser asertivos ante los comentarios técnicos de sus asistentes y que, a su vez, los estimulan para que los realicen. En la subcategoría de conocimiento denominada relación del entrenador con sus jugadores, los resultados fueron heterogéneos, pues determinados entrenadores manifestaron que algunos de sus jugadores les han aportado saberes relacionados con aspectos tácticos; sin embargo, otros expresaron que no mantienen una comunicación directa con sus jugadores y que más bien son sus asistentes los que realizan esa labor.

Referente a los resultados de la subcategoría de mentores, esta fue bien valorada, como fuente de adquisición de conocimiento, por todos los entrenadores. En términos generales, ellos manifestaron que, en su labor profesional, han tomado muchos aspectos de sus exentrenadores, y que no solo tomaban de ellos aspectos que consideraban positivos, sino también los negativos, pues los tomaban como experiencias para no aplicarlos en el ejercicio de su profesión.

La subcategoría de estudios independientes también fue altamente valorada como fuente de conocimiento, sobre todo, en los resultados del cuestionario (ver Tabla 4). Esta subcategoría, en conjunto con todas las anteriores ubicadas en educación informal, forma parte de mecanismos a través de los cuales las experiencias producen aprendizaje para el entrenador (Erickson, Bruner, MacDonald y Coté, 2008; Pérez, 2002).

Otro estudio desarrollado por Lemyre et al. (2007) menciona que la formación del entrenador no debe basarse únicamente en el conocimiento formal para aprender a dirigir o entrenar, sino que también puede apoyarse en experiencias previas del entrenador como jugador o asistente técnico y, por supuesto, en las propias experiencias de aprendizaje que subyacen en la competencia y sus reflexiones alrededor de ellas proveen aprendizajes importantes. Algo similar encontraron en su estudio Cushion, Armour y Jones (2012), quienes expresaron que la experiencia del entrenador se ubica entre sus principales fuentes de conocimiento; además, mucho de lo que aprenden es a través de cursos prácticos y de su interacción con su contexto de entrenamiento y competición, así como de una variedad de fuentes informales de conocimiento.

Prosiguiendo con el tema de la educación informal, estudios realizados por Abraham, Collins y Martindale (2006), Nash y Collins (2006), Erickson et al. (2008) y Pérez (2002) concluyeron que la experiencia profesional es una de las fuentes de información más importantes para el desarrollo del entrenador deportivo.

Por último, la interacción entre colegas se ubicó, en los resultados del cuestionario, en el último lugar del puntaje promedio (ver Tabla 4) de todas las subcategorías de la categoría educación informal. También, en las entrevistas, 8 de los 10 entrenadores expresaron no tener relación o vínculos que les permitieran compartir conocimientos con sus colegas. Un estudio realizado por Cushion et al. (2012) manifiesta que, en la última década, muchas investigaciones han demostrado que diversos tipos de interacción con otros colegas es la principal fuente de conocimiento. Sin embargo, esto no coincide con los resultados de este estudio; quizás este fenómeno se deba a aspectos educativos y culturales propios del contexto en el cual se desenvuelven los entrenadores. 
Prosiguiendo con la categoría de educación informal, autores como Cushion et al. (2012), Nasch y Collins (2006), Lemyre et al. (2007), Werthner y Trudel (2006) expresan que la toma de decisiones acertadas durante el proceso de entrenamiento y competición está sustentada, fundamentalmente, en la educación informal, es decir, el aprendizaje empírico.

Ahora bien, en la categoría de conocimiento relacionada con la educación formal, específicamente lo que en la entrevista se denominó educación continua, todos los entrenadores manifestaron que esta era importante, para el ámbito del entrenamiento más que para el de dirigir. Por el lado de los resultados del cuestionario, se puede notar, observando la Tabla 4, que esta categoría no fue ubicada por los entrenadores como una de las más importantes respecto a la adquisición de conocimiento para el ejercicio de su profesión, pues, después de la subcategoría de interacción entre colegas, fue la que obtuvo el menor puntaje.

El tema de la educación formal, en el cual los entrenadores manifestaron que todo lo que abarca la educación continua les ha servido poco para su labor de dirigir en la competencia, se ve reforzado por estudios realizados por Cushion et al. (2012), Nasch y Collins (2006), Lemyre et al. (2007), Werthner y Trudel (2006). Ellos encontraron que la toma de decisiones de los entrenadores se basa en procesos informales de educación, es decir, en situaciones de aprendizaje empíricas.

Otra investigación realizada por Castillo, Ramis, Cruz y Balaguer (2015) encontró que los entrenadores, luego de recibir un taller sobre coaching, expresaron que, al final de este, se sentían preparados para integrar todos los conocimientos adquiridos a sus prácticas de entrenamiento. Esto es congruente con lo que expresaron los entrenadores del presente estudio, cuando manifestaron que todos los conocimientos dados por los diversos escenarios de la educación continua les ayudaban a la planificación de los entrenamientos.

Por tanto, si analizamos a profundidad los resultados que se dieron en las categorías de educación formal e informal, se podría afirmar que los conocimientos para dirigir y entrenar están ligados a aspectos cognitivos, adquiridos anteriormente en la experiencia como futbolista, estudios independientes y el aprendizaje subyacente al realizar el ejercicio mental de estar planteando partidos, más que de los saberes de índole académica.

La categoría de conocimiento denominada recursos tecnológicos, específicamente la subcategoría de manejo de herramientas tecnológicas, resultó, en la aplicación del cuestionario, con uno de los puntajes más altos para el ámbito de dirigir, mientras que para el ámbito de entrenar se ubicó en la tercera casilla (ver tabla 4) de mayor puntaje. Respecto a la aplicación de las entrevistas, en términos generales, los entrenadores manifestaron que la puesta en práctica de las herramientas tecnológicas, por un lado, les facilitaba la comunicación para transmitir el mensaje táctico y estratégico hacia sus futbolistas y, por el otro, también les ayudaba en la planificación de los entrenamientos.

Pareciera que los resultados anteriores dejan evidenciar que los avances tecnológicos van teniendo cada vez más vigencia e importancia para el ejercicio práctico profesional del entrenador de fútbol. La información que brindan las herramientas tecnológicas está siendo utilizada por los entrenadores como criterios no solo para la toma de decisiones en la planificación del entrenamiento, sino también en el ámbito competitivo.

\section{Conclusiones}

De acuerdo con los resultados obtenidos del estudio, tanto en las entrevistas como en los cuestionarios, se observó que en todas las categorías con sus respectivas subcategorías de adquisición de conocimientos -excepto en la interacción entre colegas- los entrenadores manifestaron haber obtenido conocimiento para dirigir y entrenar, no obstante, pareciera que la experiencia como jugador y el manejo de herramientas tecnológicas son las subcategorías que brindan el mayor conocimiento a los entrenadores del estudio. Sin embargo, la interacción entre colegas fue la que obtuvo un menor valor en la adquisición de conocimiento.

También, se puede dilucidar, a través del análisis del estudio, que la formación del entrenador de fútbol no solo es compleja, sino que impera en ella una diversidad de fuentes de adquisición de conocimientos. Por 
tanto, los resultados de la investigación inducen a reflexionar sobre las diversas pautas curriculares que se deben seguir en la futura formación de entrenadores de fútbol élite, pues pareciera evidente que utilizar la metodología del sistema educativo tradicional no es lo más conveniente; esta no facilita ni promueve una adquisición de destrezas prácticas para entrenar y dirigir los equipos (Jiménez-Sáiz, Lorenzo-Calvo y Gómez-Ruano, 2009).

\section{ReFERENCIAS}

Ayala-Zuluaga, C. F., Aguirre-Loaiza, H. H. y Ramos-Bermúdez, S. (2015). Formación académica y experiencia deportiva de los entrenadores suramericanos. Revista Brasileira de Ciências do Esporte, 37(4), 367-375. DOI: http://dx.doi.org/10.1016/j.rbce.2015.09.001

Abraham, A., Collins, D. y Martindale, R. (2006). The coaching schematic: Validation through expert coach consensus. Journal of Sports Sciences, 24(6), 549-564. DOI: 10.1080/02640410500189173

Barros, G. C., Ribeiro, I. M., Arroyo, M. P. M., Rosado, A. F. B., Sousa, T. M. T. y Pereira, P. F. S. (2010). Autopercepción de las competencias profesionales de los entrenadores de fútbol en función de la experiencia profesional y de la formación académica. Cuadernos de Psicología del Deporte, 10(1). Recuperado de http:// revistas.um.es/cpd/article/view/110261/104901

Bisquerra, R. y Pérez-Escoda, N. (2015). ¿Pueden las escalas Likert aumentar en sensibilidad? REIRE Revista d'innovació i recerca en educació, 8(2), 129-147. DOI: 10.1344/reire2015.8.2.828

Castillo, I., Ramis, Y., Cruz, J. y Balaguer, I. (2015). Formación de Entrenadores de Fútbol Base en el Proyecto PAPA. Revista de Psicología del Deporte, 24(1), 131-138. Recuperado de http://web.a.ebscohost.com.una.idm.oclc.org/ehost/pdfviewer/pdfviewer?sid=0c7cle47e3b6-4859-a6a6-659ea8172b05\%40sessionmgr4007\&vid=1\&hid=4207

Coté, J., Salmela, J. H., Trudel, P., Baria, A. y Russel, S. (1995). The coaching model: a grounded assessment of expert gymnastic coaches knowledge. Journal of Sport \& Exercise Psychology, 17(1), 1-17. DOI: http:// dx.doi.org/10.1123/jsep.17.1.1

Coté, J., Young, B., North, J. y Duffy, P. (2007). Towards a definition of excellence in sport coaching. International Journal of Coaching Science, 1, 3-17. Recuperado de https://www.researchgate.net/ publication/43501231_Towards_a_Definition_of_Excellence_in_Sport_Coaching

Cushion, C., Armour, K. y Jones, R. (2012). Coach education and continuing professional development: experience and learning to coach. Quest, 55(3), 215-230. DOI: http://dx.doi.org/10.1080/00336297.2003.10491800

Erickson, K., Bruner, M., MacDonald, D. y Coté, J. (2008). Gaining Insight into Actual and Preferred Sources of Coaching Knowledge. International Journal of Sports Science \& Coaching, 3, 4, 527-538. DOI: https:// doi.org/10.1260/174795408787186468

Feu-Molina, S., Ibáñez-Godoy, S. J., Lorenzo-Calvo, A., Jiménez-Saiz, S. y Cañadas-Alonso, M. (2012). El conocimiento profesional adquirido por el entrenador de balonmano: experiencias y formación. Revista de psicología del deporte, 21(1), 107-115. Recuperado de http://www.redalyc.org/html/2351/235124455014/

Jiménez-Sáiz, J., Lorenzo-Calvo, A., Gómez-Ruano, M. y Lorenzo-Calvo, J. (2009). La adquisición del conocimiento en entrenadores expertos en baloncesto. Apuntes educación física y deportes, 2(96), 47-52. Recuperado de http://abacus.universidadeuropea.es/bitstream/handle/11268/601/385.pdf?sequence=1\&isAllowed=y

Jiménez-Saiz, S., Gómez-Ruano, M. Á., Borrás-Luján, P. y Calvo, A. L. (2007). Factores que favorecen el desarrollo de la pericia en entrenadores expertos en baloncesto. Cultura, Ciencia y Deporte, 2(6), 145-149. Recuperado de http://www.redalyc.org/pdf/1630/163017580007.pdf

Lemyre, F., Trudel, P. y Durand-Bush, N. (2007). How Youth-Sport Coaches learned to coach. The Sport Psychologist, 21, 191-209. Recuperado de http://citeseerx.ist.psu.edu/viewdoc/download? doi=10.1.1.463.5911\&rep=rep1\&type=pdf

Nash, C. y Collins, D. (2006). Tacit Knowledge in expert coaching: Science or art? Quest, 58(4), 465-477. DOI: http://dx.doi.org/10.1080/00336297.2006.10491894 
Okuda-Benavides, M. y Gómez-Restrepo, C. (2005). Métodos en investigación cualitativa: triangulación. Revista Colombiana de Psiquiatría, 24(1), 118-124. Recuperado de http://www.scielo.org.co/scielo.php? script=sci_arttext\&pid=S0034-74502005000100008

Patton, M. Q. (2002). Qualitative evaluation and research methods. 3th ed. Newbury Park, CA: Sage.

Pérez, C. (2002). Caracterización del entrenador de alto rendimiento deportivo. Cuadernos de Psicología del Deporte, 2(1), 15-37. Recuperado de https://digitum.um.es/xmlui/bitstream/10201/27326/1/Caracterizaci\%C3\%B3n \%20del\%20entrenador\%20de\%20alto\%20rendimiento\%20deportivo.pdf

Rosado, A., Palma, N., Mesquita, I. y Moreno, P. (2007). Percepción de los jugadores de fútbol, de distinto nivel, sobre sus entrenadores. Revista de Psicología del Deporte, 16(2), 151-165. Recuperado de http://www.rpdonline.com/article/viewFile/18/18

Vallée, C. N. y Bloom, G. A. (2005). Building a successful university sport program: key and common elements of expert coaches. Journal of Applied Sport Psychology, 17(3), 179- 196. DOI: 10.1080/10413200591010021

Vinicia, J., Mayorga, D., Ruiz, J. y Blanco, H. (2016). La comunicación educativa de entrenadores de fútbol en competición. Federación Española de Asociaciones de Docentes de Educación Física, (29), 17-29. Recuperado de http://web.a.ebscohost.com.una.idm.oclc.org/ehost/pdfviewer/pdfviewer? sid=bf7be36c-e6bc-4690-a1a5-4b225b933b01\%40sessionmgr4008\&vid=1\&hid $=4207$

Werthner, P. y Trudel P. (2006). A New Theoretical Perspective for Understanding How Coaches learning to coach. The Sport Psychologist, 20, 198-212. DOI: http://dx.doi.org/10.1123/tsp.20.2.198

\section{BY-NC-ND}

